\title{
Repensando la confianza como factor crítico en la gestión organizativa
}

\section{Rethinking trust as a critic factor in the organizational behaviour}

\author{
Manuel Guillén Parra ${ }^{1}$ \\ Álvaro Lleó de Nalda ${ }^{2}$ \\ Gines Santiago Marco Perles ${ }^{3}$ \\ Universidad de Valencia / Universidad Politécnica de Valencia (España) \\ Universidad Católica de Valencia (España)
}

Recibido el 15 de abril de 2010 y aceptado el 15 de octubre de 2010

$\mathrm{N}^{\circ}$ de clasificación JEL: M14

DOI: $10.5295 /$ cdg. $100228 \mathrm{mg}$

\section{Resumen:}

La confianza en el mundo de los negocios es un factor esencial que viene siendo estudiado por la literatura del Management desde hace décadas. Partiendo de la propuesta seminal de Mayer et al.(1995), acerca de qué es la confianza y cómo se desarrolla en el seno de las organizaciones, proponemos trazar una crítica constructiva al modelo descrito a partir de las aportaciones de Spaemann, autor de dilatada trayectoria en el marco de la denominada "Filosofía Moral de la Europa Continental". A partir de esta crítica constructiva propondremos una revisión del modelo y sugeriremos algunas matizaciones. Defendemos aquí que las mejoras del modelo permitirán explicar el fenómeno de la confianza de un modo más preciso y acorde al comportamiento humano gracias a la síntesis humanista que aporta el diálogo entre ética y ciencias sociales.

Palabras clave:

confianza organizativa, integridad, ética, ética integral.

\begin{abstract}
:
Trust in business is an essential factor that has been being studied by the Academy for decades. From the seminal proposal of Mayer et al. (1995) about the concept of trust and how it is developed inside organizations we propose a revision of the model described by the contributions of Spaemann, author of expanded trajectory within the framework of the denominated "Moral Philosophy of Europe Continental". From this constructive critic we defend here that the improvements of the model will allow explaining the phenomenon of trust generation with
\end{abstract}

\footnotetext{
${ }^{1}$ Director del Instituto para la Ética en la Comunicación y en las Organizaciones (IECO-FUNDACION COSO). c/Mar 26, $2^{\circ}$ pta 4. 46003 Valencia.

${ }^{2}$ Investigador del Instituto para la Ética en la Comunicación y en las Organizaciones (IECO-FUNDACION COSO). c/Mar 26, $2^{\circ}$ pta 4. 46003 Valencia.

3 Universidad Católica de Valencia, Dpto. de Ética y Deontología Profesional, Campus de San Juan Bautista, C/ Guillem de Castro, 175. 46008 Valencia. Email: Gines.Marco@ucv.es
} 
more precision and agreed to the human behavior by the incorporation a humanist synthesis which contributes to the dialogue between ethics and social sciences

\section{Keywords:}

organizational trust, integrity, ethics, integral ethics. 


\section{INTRODUCCIÓN}

La confianza en el ámbito empresarial es uno de los elementos que viene siendo estudiado por la literatura del Management desde hace décadas. Partiendo de una concepción de la "empresa" como organización humana ${ }^{1}$, el fenómeno de la interdependencia entre sus miembros es esencial en la actividad diaria: trabajar con otras personas en una organización implica la interdependencia para poder conseguir los objetivos propios y los de la organización (Jensen y Mecking, 1976; Meyer, 1983; Sitkin y Bies, 1994; Williamson, 1975). Esta interdependencia lleva consigo dos aspectos importantes; por un lado, implica riesgos, ya que, precisamente, se condiciona el actuar propio al de otras personas. Por otro lado, se quiere potenciar la cooperación para que los sistemas de trabajo que requieren interdependencia -como el trabajo en equipo o la gestión participativa (Marin-Garcia, 2002)- resulten efectivos. Además, valores como la capacidad de motivación, el liderazgo, la promoción de las personas en la organización y otros, son intangibles incuestionables que se desarrollan en el seno de la interdependencia.

En el sentido de lo expuesto, la confianza organizativa resulta crucial al hablar de la interdependencia. Ya sea para asumir los riesgos, ya sea para atender al propósito de la cooperación en el trabajo, ya sea para potenciar el desarrollo de valores intangibles, en todos estos casos se precisa una comprensión profunda del fenómeno de la confianza interpersonal.

Esta es precisamente la línea de investigación que Mayer, Davis y Schoorman abordaron en su artículo "An integrative model of organizacional trust" en 1995. Su objetivo era el de profundizar en el estudio de la confianza interpersonal, detectar los elementos y las relaciones existentes entre los implicados en la relación, con el propósito de entender el fenómeno de la generación de confianza en el ámbito de las organizaciones.

Mayer et al. (1995) entienden la confianza interpersonal como "el hacerse vulnerable" al decidir hacerse dependiente de la otra persona, con lo que al confiar se asume un riesgo (cfr. Mayer et al., 1995:712). Para que se produzca la confianza, debe tenerse en cuenta a las partes implicadas: tanto a la persona que confía (el depositante de la confianza), como la persona en la que se confía (el depositario de la confianza). Además, es necesario contemplar otros elementos que intervienen en el proceso. Entre estos elementos se encuentran las características de las personas que influyen en la generación de confianza. Por parte del depositante está su "propensión a confiar", es decir, su disposición a asumir el riesgo de confiar en ese momento. Por parte del depositario está su "confiabilidad", es decir, la capacidad de generar una confianza en el depositante. El depositante evaluará la confiabilidad del depositario en función de tres características: su habilidad, su benevolencia y su integridad. Mayer et al., al describir estos tres antecedentes de la confianza, afirman en una nota explicativa que existe un paralelismo entre estas tres dimensiones y los tres elementos, que según la Retórica de Aristóteles, marcan una comunicación persuasiva: el logos (habilidad), el pathos (benevolencia) y el ethos (integridad).

Esta propuesta seminal acerca del concepto de la confianza y de cómo se genera en el seno de las organizaciones ha supuesto un importante avance en la comprensión del

\footnotetext{
1 "un conjunto de personas, que se unen para conseguir unos objetivos comunes, mediante unos medios, tangibles o intangibles, de modo más o menos estable" (Guillen, 2006:65)
} 
fenómeno. No obstante, y como expondremos a lo largo de este trabajo, en la propuesta de Mayer et al., se echa en falta una reflexión más profunda acerca de la dimensión ética de la confianza y una mención explícita a la libertad humana, que es precisamente la que justifica la presencia de dicha dimensión.

En este trabajo nos proponemos trazar una crítica constructiva al modelo descrito por Mayer, Davis y Schoorman a partir de las aportaciones de la obra de Spaemann y su consideración de la ética y la libertad humana al describir el fenómeno de la confianza. En este sentido, propondremos una mejora del modelo a partir de un marco holístico e integral que englobe tanto los aspectos técnicos como los aspectos éticos en la explicación del fenómeno de la confianza interpersonal en las organizaciones.

Para llevar a cabo este objetivo, este trabajo se estructurará en cuatro partes. En la primera parte se describe con más detalle el modelo de generación de confianza propuesto por Mayer, Davis y Schoorman, uno de los modelos teóricos de más amplio reconocimiento en la literatura de Management. En la segunda parte se describen las limitaciones de este modelo, derivadas de una concepción limitada de la dimensión ética de la confianza. En la tercera parte, y para superar estas limitaciones expuestas, se propone la incorporación de las aportaciones del filósofo alemán Robert Spaemann en su descripción de la confianza. Finalmente, se propone un concepto holístico de confianza en el que confluyan de modo explícito los aspectos técnicos (el proceso de generación de confianza propuesto por Mayer et al. 1995) y los éticos (con la mención explícita de los elementos que sugiere Spaemann).

\section{DESCRIPCIÓN DEL MODELO DE GENERACIÓN DE CONFIANZA DE MAYER, DAVIS Y SCHOORMAN}

Mayer, Davis y Schoorman, en su artículo titulado An integrative model of organizational trust (1995), definen la confianza interpersonal en el seno de las organizaciones como "la decisión de la persona que confía (el depositante de la confianza) de hacerse vulnerable ante una acción de otro (el depositario de la confianza)". Esta decisión está basada en la suposición de que el depositario de la confianza hará bien esa acción sin que se le controle, precisamente porque se confía en él o ella (cfr. Mayer et al., 1995:712).

La propuesta de estos autores es acorde a otras definiciones de confianza organizativa que relacionan este concepto con la idea de que una persona se hace vulnerable al depender de otra a la que no se controla (Butler, Jr. y Cantrell, 1984; Deutsch, 1958; Knox y Kee, 1970; Zand, 1972).

La elección de estos autores como referente para el estudio de la confianza se ha tomado por tres razones fundamentales: en primer lugar, porque ofrecen una síntesis transversal de toda la investigación hecha hasta ese momento acerca de la confianza organizativa. En segundo lugar, porque su obra destaca por la claridad con la que se define el concepto de confianza, y por el modo en que se distinguen y se fijan las relaciones entre los distintos elementos que intervienen en el proceso de su generación. En tercer lugar, nos encontramos ante un trabajo académico publicado en una de las publicaciones de más prestigio en el área de Management y el artículo en particular cuenta un con alto impacto (en la fecha de cierre de este trabajo constaba de más de 4000 citas en Google Schoolar), lo que permite calificarlo de artículo seminal sobre la confianza organizativa. 
A nuestro juicio, lo que aporta la definición de estos autores es precisamente su descripción del acto de confiar como "decisión de hacerse vulnerable" frente a otra persona. Como veremos después, en el modelo no se hace explícito que se trata de una decisión no condicionada, de una decisión humana libre, que estará influenciada por unos antecedentes, pero que precisamente por ser libre es impredecible, de ahí la necesidad de hablar de riesgo, y de juicio acerca de aspectos morales o éticos de los agentes que intervienen en la relación.

Figura 1

modelo de confianza.

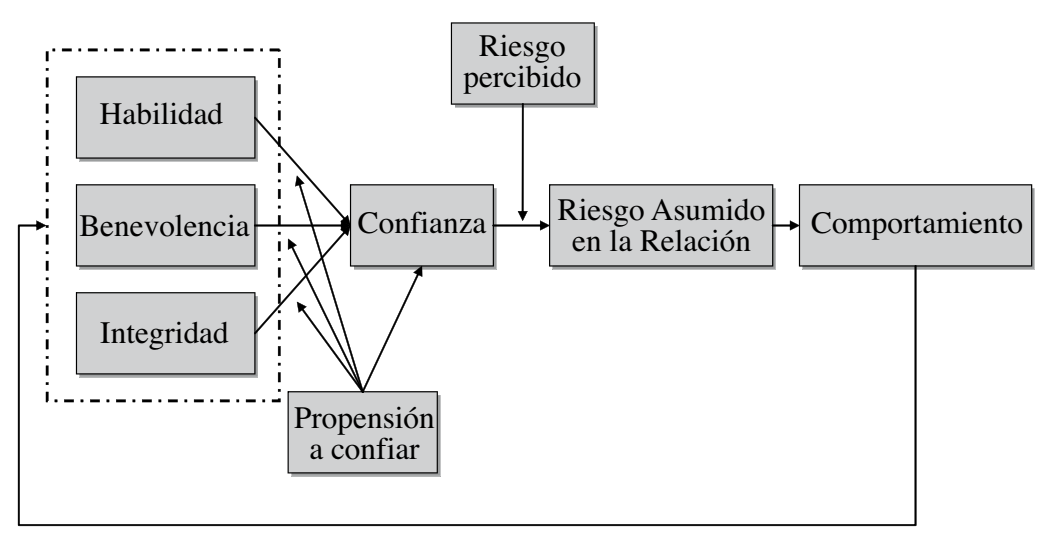

Fuente: Traducción de (Mayer et al., 1995:715)

Como se puede apreciar en la Figura 1, los autores distinguen varios elementos en el proceso de generación de confianza. En síntesis, podemos hablar de tres elementos que la explican. En primer lugar aparecen los agentes que entran en juego en cualquier acción de confiar: el depositante y el depositario de la confianza. En segundo lugar, se distinguen las características de los agentes que hay que tener en cuenta para valorar si se genera confianza o no, es decir, los factores que inciden en la generación de confianza (tanto en el depositante, como en el depositario). Y, en tercer lugar, se distingue el concepto de riesgo asumido como resultado de confiar. Estos tres elementos son descritos como componentes de un proceso dinámico. Explicaremos a continuación con más detalle este proceso.

Mayer et al. (1995) dirán que la confianza depende tanto de la persona en la que se va confiar como de la persona que confía. El depositante, influye en el proceso de generación de confianza mediante su propensión a confiar, es decir, su tendencia a asumir o no riesgos. En este sentido, si una persona tiene tendencia a no asumir riesgos, es difícil que confíe aunque se den otros factores. Este elemento constituye una condición necesaria, pero no suficiente, para que se inicie una relación de confianza entre dos personas. Complementariamente, el depositario (la persona en la que se va a confiar) desempeña un papel crucial, pues su confiabilidad será evaluada por el depositante, es decir, juzgará si es digno de confianza o no. Su confiabilidad será evaluada mediante el juicio de una serie de caracte- 
rísticas que Mayer et al. (1995) agrupan en tres categorías: su habilidad, su benevolencia y su integridad. A continuación, explicaremos brevemente estas tres categorías:

- La habilidad es entendida como el conjunto de conocimientos, destrezas y competencias del depositario que le capacitan para desempeñar una función determinada (cfr. Mayer et al., 1995:717). Muchos otros autores definen esta categoría con el término competencia (Butler, Jr., 1991; Butler, Jr. y Cantrell, 1984; Knox y Kee, 1970; Mayer et al., 1995). Es un elemento de carácter situacional, ya que se tiene habilidad para realizar una actividad determinada, con lo que la confianza en esta categoría depende del contexto en el que se desarrolla la acción. Por ejemplo, si se plantea la posibilidad de confiar en una persona para que decida sobre la conveniencia de instalar una red wifi en la planta de producción, esta persona deberá tener una serie de conocimientos técnicos (o deberá tener la formación suficiente para adquirirlos) para que se pueda confiar en ella.

- La benevolencia es entendida como el conjunto de motivos e intenciones que persigue el depositario de la confianza en esa acción concreta. Lo que se evalúa es si el depositario busca el beneficio del depositante y no únicamente su beneficio propio. Esta buena intención presupone, a juicio de los autores, que existe algún tipo de vínculo entre los implicados en la relación, que es el que les lleva a no disfrazar, so capa de confianza, una conducta oportunista. Como dicen (Mayer et al., 1995:719) "la benevolencia sugiere que el depositario tiene algún tipo de relación -atadura- con el depositario".

- La integridad es definida por estos autores como aquellos principios que rigen el comportamiento del depositario. El depositante de la confianza evaluará si comparte o no estos principios con el depositario de la confianza antes de asumir el riesgo de confiar.

Los autores defienden que, aunque se puedan distinguir los antecedentes de la confianza en tres tipos o categorías, se requiere de todos ellos para que ésta se produzca. Únicamente después de que el depositante de la confianza haya realizado una evaluación de estos tres tipos de factores, se podrá establecer el nivel de confianza que el depositario suscita en el depositante.

Mayer et al. (1995), en una nota a pie de página de este artículo seminal al que venimos refiriéndonos, describen de modo muy somero el paralelismo de los tres antecedentes de la confianza con los tres elementos que según Aristóteles debe reunir una buena comunicación. Estos tres elementos (logos, pathos y ethos) son aquellos en los que el emisor será evaluado por los receptores de su discurso.

Como es bien sabido, Aristóteles distingue en su Retórica estos tres elementos. El logos (palabra, idea) hace referencia a la argumentación del discurso del orador. Se refiere tanto los principios de los que se parte como a las relaciones causales que se van estableciendo a lo largo del proceso discursivo. En definitiva, un orador conectará mejor con sus interlocutores si es capaz de hilar su discurso con cierta competencia o saber hacer práctico.

Junto a la dimensión racional, el pathos (sentimiento, pasión) hace referencia a la emoción que pone el orador en su discurso a través del tono de voz y del lenguaje no verbal. Esta pasión es capaz de despertar una respuesta similar en los oyentes de modo que el receptor haga propio el mensaje transmitido y se establezca un vínculo entre ellos. Aristóteles dirá que el pathos afecta al logos ya que la pasión complementa a una exposición clara y ordenada de las ideas. 
Por último, el ethos (carácter, ética), hace referencia a la honradez del emisor. Esta honradez es capaz de incrementar la capacidad persuasiva del discurso ya que, como dice Aristóteles "a los hombre buenos los creemos de modo más pleno y con menos vacilación... No es verdad, como suponen algunos escritores en sus tratados de retórica, que la bondad personal de quien habla no contribuye nada a su poder de persuasión; por el contrario, su carácter, casi puede considerarse el medio más efectivo de persuasión que posee" (Aristotle, 1992).

Como hemos dicho, Mayer et al. (1995:717), hacen referencia a la existencia de un paralelismo entre los tres elementos de la comunicación persuasiva de Aristóteles y la confianza. El logos sería identificado con la competencia o habilidad del depositario de la confianza, el pathos con su benevolencia y el ethos con su integridad.

La confiabilidad del depositario es evaluada considerando estas tres dimensiones, y tras hacerlo, el depositante deberá tomar la decisión de hacerse vulnerable o no. Esta acción viene reflejada en el modelo mediante la variable "riesgo asumido en la relación", que es la manifestación de la confianza en su comportamiento. De este modo, el riesgo asumido por el depositante ha de ser proporcional a la confianza que tiene que suscitar el depositario. Por poner un ejemplo, no es lo mismo decidir sobre si le dejo mi bicicleta a alguien, que sobre si le dejo los resultados de una investigación en la que he invertido gran cantidad de tiempo y esfuerzo, ya que las posibles consecuencias negativas en ambas acciones no tienen las mismas repercusiones.

Finalmente, conviene subrayar que el modelo propuesto ya en 1995 entiende el proceso de generación de confianza como un proceso gradual y dinámico. El depositante reconsiderará el juicio sobre la confiabilidad del depositario al observar su comportamiento.

Con posterioridad a la publicación del modelo en el año 1995, Schoorman, Mayer, and Davis publicaran otro trabajo en 2007 con el título: "An integrative model of organizacional trust: past, present and future". En él se recogen los comentarios que han surgido en los doce años posteriores a la aparición de su artículo seminal, así como los nuevos campos en los que sigue abierta la investigación. El tenor de tales comentarios apunta a las siguientes claves:

En primer lugar, los autores reconocen la necesidad de ampliar la investigación del modelo en otros niveles de análisis más amplios que el estrictamente interpersonal (confianza en el grupo, confianza en la organización...) para poder diseñar así un modelo más generalizable. En segundo lugar reconocen la necesidad de seguir estudiando con más profundidad la variación de la generación de confianza a lo largo del tiempo y la importancia de ampliar el estudio acerca de la confianza como relación mutua y/o recíproca. En tercer lugar, apuntan la conveniencia de profundizar en la relación entre confianza, riesgo asumido y sistemas de control. Y por último subrayan la conveniencia de mejorar la escala de medida de la variable confianza².

\footnotetext{
2 Tras el estudio de la obra completa de Mayer et al. (1995) y de todos los trabajos empíricos que han realizado con su modelo (Mayer y Davis, 1999; Mayer y Gavin, 2005; Schoorman et al., 1996; Schoorman y Ballinger, 2006; Serva et al., 2005), la escala para medir la variable confianza es la que más modificaciones ha sufrido. Con la escala de 1996 se obtuvo un alpha de conbrach de 0,60 . Tras diversas modificaciones se ha llegado a un alpha de conbrach de 0,84 con la escala de Schoorman y Ballinger (2006).
} 
Con estas sugerencias, los autores del modelo reconocen algunas de las limitaciones de su trabajo inicial. Junto a estos aspectos que deben seguir siendo estudiados existen otros como: el papel de los afectos y la emociones en la generación de confianza, la ruptura y la reconstrucción de la confianza, el papel de las distintas culturas en la generación de confianza y la generación de confianza en contextos específicos viendo el efecto que producen ciertas variables contextuales (Schoorman et al., 2007). Lo que aquí proponemos se plantea precisamente en línea con algunas de estas sugerencias descritas. En concreto, nuestras aportaciones van ligadas a la segunda y cuarta claves.

\section{LIMITACIONES DEL MODELO DE GENERACIÓN DE CONFIANZA DE MAYER ET AL.}

El modelo de confianza propuesto por Mayer, Davis y Schoorman merece un reconocimiento, no sólo por su claridad y por haber logrado una sistematización de todas las investigaciones precedentes relacionadas con el concepto y el proceso de generación de confianza, sino también, y sobre todo, por su audacia a la hora de invocar un concepto como el de la confianza, que por constituir un activo intangible, nunca podrá ser objeto directo de observación y experimentación en todas sus dimensiones. No obstante, queremos subrayar ahora que esta propuesta adolece de ciertas limitaciones, inherentes al esquematismo de todo modelo, y que podrían ser superadas en parte con algunas matizaciones.

En primer lugar, la dimensión ética que estaría detrás de la noción de integridad propuesta por estos autores alude a una serie de principios a los que se adhiere el depositario -que no se llegan a explicitar- y que el depositante considera aceptables, con lo que se podría decir que los comparte. Esta concepción de integridad nos parece incompleta, ya que deja de lado la interiorización práctica de estos principios hasta llegar a constituirse en hábitos buenos del carácter (virtudes). Las virtudes morales (como son la sinceridad, la generosidad, la equidad y tantas otras) constituyen un elemento observable del comportamiento humano sobre las que se emiten juicios acerca de la integridad de las personas. Alguien puede decir que se adhiere a unos principios morales, pero después no cumplirlos de modo ordinario, precisamente por falta de virtud.

A diferencia de lo que defienden Mayer et al. (1995), parece razonable afirmar que lo primero que conforma el juicio sobre la integridad del depositario es su carácter, que queda reflejado en su comportamiento. A partir de la observación de los hábitos del carácter, el depositante podrá juzgar la integridad del depositario. Sólo después de que el depositario infunda confianza con su actuar, el depositante se interesará por descubrir los principios que rigen su obrar, y por confirmar que son los que guían su conducta.

Además, los hábitos del carácter ${ }^{3}$, no sólo constituyen el primer elemento para poder evaluar la integridad del depositario sino que conforman la verdadera garantía de ésta. Si el depositario no demostrara con los hechos los principios a los que dice adherirse, lo que suscitaría sería un rechazo en el depositante ya que pensaría que es un hipócrita o, al menos, que dice una cosa y luego hace otra. De este modo, más le valdría al depositario no

${ }^{3}$ En este caso hablamos de hábitos operativos buenos, es decir, de las virtudes. 
decir nada acerca de sus principios, y más en temas relacionados con la ética, dimensión de la conducta que mira en primer lugar el modo de obrar bueno o malo.

La segunda limitación que vemos en este modelo también hace referencia a la dimensión de la integridad como elemento constitutivo de la confiabilidad del depositario. La integridad de una persona no es sólo el resultado de una adhesión a principios intelectuales, ni tampoco lo es sólo a la corroboración de un carácter y unas virtudes morales. Junto a ambas cosas, la persona íntegra es aquella que sabe juzgar cómo obrar bien en situaciones particulares, lo que requerirá de la habilidad para establecer juicios acerca de lo bueno y lo malo en cada situación concreta. Esta capacidad de juicio es definida por los clásicos precisamente como la virtud de la prudencia, que es moral e intelectual a la vez.

Del mismo modo que cabe hablar de la capacidad para tomar decisiones para alcanzar unos resultados haciendo un uso adecuado de los recursos (eficiencia)...es posible hablar de la capacidad de tomar decisiones de modo ético. No es lo mismo juzgar acerca de la utilidad de una acción que de su conveniencia (un expediente de regulación de empleo puede ser una fórmula muy eficiente desde el punto de vista exclusivamente de la utilidad pero que tendría que someterse a un juicio ético para calificarse de conveniente).

Como expone Melé (2000: 420) “el ser humano posee una única potencia o facultad a la que solemos denominar inteligencia o razón, pero puede tener distintos usos o formas de empleo. Uno de ellos está orientado a la transformación racional del mundo exterior. Es una racionalidad instrumental, adecuada para lograr resultados...La racionalidad instrumental está, pues, ordenada a la eficacia, pero eso no es suficiente... Se precisa otro modo de racionalidad que guíe la racionalidad instrumental hacia lo conveniente. A esa racionalidad que dirige la acción hacia lo verdaderamente conveniente se la puede denominar racionalidad directiva".

La racionalidad directiva a la que hace referencia Melé se identifica con la virtud de la prudencia de la que habla Aristóteles "La acción es regida por otra disposición racional distinta de la técnica que denomina prudencia, que la define del siguiente modo: La prudencia es una disposición racional verdadera y práctica respecto de lo que es bueno para el hombre....al decidir no sólo se ocasionan resultados externos, sino que también afecta al que decide. Al decidir no sólo elegimos qué queremos hacer, sino también qué clase de persona queremos ser" (Mele, 2000 :421). De este modo, la persona íntegra es aquella que, además de unos principios y unos hábitos del carácter, cuenta con la habilidad o la capacidad de tomar buenas decisiones en sentido ético.

Esta capacidad de juicio es un factor que debería recogerse dentro de la dimensión de la integridad del depositario. En la medida en que lo que se pone en juego es algo más personal, más pesará la capacidad de juzgar bien o virtud de la prudencia del depositario, ya que la confianza tendrá un carácter más personal que técnico (más de saber acertar que de saber hacer).

La tercera limitación que encontramos en la propuesta de Mayer et al. (1995), es de carácter más global, y hace referencia a los planteamientos teóricos que sostienen su concepto de confianza. Esta limitación se desprende del análisis del modelo en su conjunto. Se trata de un modelo explicativo del comportamiento humano que, sin embargo deja escaso protagonismo a la libertad humana. Mayer et al. (1995) aluden a "la decisión de hacerse vulnerable" por parte del depositante de la confianza, como elemento consustancial en toda 
disposición a confiar. No obstante, la reacción no siempre predecible del depositario de la confianza (precisamente por tratarse de un sujeto libre) no es nítidamente mencionada.

Si existe un riesgo en la confianza es precisamente porque tanto el que confía como la persona en la que se confía son sujetos libres. Porque el depositante es libre, cabe la alternativa de confiar o no hacerlo, y porque el depositario es libre, cabe la posibilidad de que actúe de un modo u otro. Esto no quiere decir que nos hallemos ante una incertidumbre permanente ni absoluta. Cabe cierta previsión ya que cualquier persona, posee una naturaleza humana que le dispone a obrar habitualmente de un determinado modo, es decir, algo que le inclina a actuar de un modo y no de otro en una determinada situación.

Esta referencia a la naturaleza humana y a su condición libre como base para entender los dinamismos del comportamiento es clave si no se quiere caer en una visión excesivamente mecanicista del comportamiento humano en la que el concepto de libertad no tendría cabida...

Como veremos a continuación, las reflexiones acerca del concepto de confianza llevadas a cabo por Robert Spaemann (2005) son las que sugerimos como base para la mejora del modelo de Mayer et al. (1995).

\section{APORTACIONES AL CONCEPTO DE CONFIANZA A PARTIR DEL PENSAMIENTO DE SPAEMANN}

A pesar del "salto de escenario" que supone comparar un trabajo propio del ámbito de la filosofía (Spaemann, 2005) con otro del ámbito de las ciencias sociales (Mayer, et al, 1995), veremos a continuación el claro paralelismo entre ambos trabajos, y a la vez la oportunidad de utilizar las aportaciones del filósofo alemán para completar el modelo de Mayer et al. (1995) superando las limitaciones comentadas.

En línea con el pensamiento de Mayer et al (1995), Robert Spaemann dirá que "la confianza no puede mostrarse de otra forma más que haciéndose vulnerable el que confía" (Spaemann, 2005:140). Esta descripción de la confianza es prácticamente igual a la descrita por Mayer et al, (1995) con la diferencia de que incorpora de modo explícito la voluntariedad en la acción de confiar. La confianza es entendida como "un acto humano que referimos a personas, por tanto a sujetos libres" (Spaemann, 2005:136). Porque existe la libertad, confiar es siempre asumir un riesgo.

Spaemann también hablará de las distintas dimensiones de la confianza. En paralelo a los tres antecedentes de la confianza de Mayer et al. (la habilidad, la benevolencia y la integridad), Spaemann dirá que "la confianza se refiere a dos contenidos: a la competencia y a las convicciones -al querer y el poder- siendo las convicciones lo más fundamental porque la competencia tomada por sí misma siempre es ambivalente... Cuando digo que las convicciones son lo más fundamental lo digo también porque una actitud ética empuja a adquirir competencia y prohíbe suponer competencia allí donde no existe." (Spaemann, 2005:146).

Como se puede apreciar, este autor pone el énfasis en el papel de la dimensión ética como motor y enlace de las demás dimensiones, además, es precisamente la presencia de la libertad moral la que permite entender el concepto de confianza más allá de la dimensión técnica de la habilidad. No sería posible hablar de benevolencia o integridad, si no se reco- 
nociera el papel que juega la libertad en el obrar humano ya que la presunción de que existe buena intención en la acción (benevolencia) presupone la posibilidad de que pueda darse también su contrario, de que haya mala intención. Y, de igual modo, la posibilidad de hacer valoraciones acerca de la mayor o menor integridad de una persona, presupone también la posibilidad de que haya ausencia de integridad, y todo ello es precisamente porque los seres humanos son agentes libres que pueden obrar bien o mal, a favor o en contra de su propio crecimiento humano.

Tabla 1

Comparación de las aportaciones sobre de confianza de Mayer et al. y Spaemann

\begin{tabular}{|l|l|l|}
\hline & \multicolumn{1}{|c|}{ Mayer, Davis y Schoorman } & \multicolumn{1}{c|}{ Spaemann } \\
\hline Concepto & $\begin{array}{l}\text { "Decisión de una persona de hacerse } \\
\text { vulnerable ante una acción de otro. } \\
\text { Esta decisión está basada en la su- } \\
\text { posición de que la otra persona hará } \\
\text { bien esa acción sin que se le controle" } \\
\text { (Mayer et al., 1995:712) }\end{array}$ & $\begin{array}{l}\text { "Abandonarse a alguien" (Spaemann, } \\
\text { 20052) }\end{array}$ \\
\hline Ámbito & $\begin{array}{l}\text { Tan sólo la confianza interpersonal en } \\
\text { el ámbito organizativo }\end{array}$ & $\begin{array}{l}\text { Confianza interpersonal y confianza } \\
\text { personal en las instituciones. }\end{array}$ \\
\hline Antecedentes & Habilidad, Benevolencia, Integridad & competencia y convicciones \\
\hline Modelo & Si & No \\
\hline $\begin{array}{l}\text { ¿Incorpora las } \\
\text { virtudes? }\end{array}$ & No & $\mathrm{Si}$ \\
\hline $\begin{array}{l}\text { ¿Incorpora la } \\
\text { prudencia? }\end{array}$ & No & $\mathrm{Si}$ \\
\hline $\begin{array}{l}\text { ¿Explicita la } \\
\text { libertad? }\end{array}$ & No & $\mathrm{Si}$ \\
\hline
\end{tabular}

Fuente: Elaboración propia

Partiendo de un contexto personal, Spaemann traslada sus aportaciones al ámbito de las organizaciones, algo que no se da en el caso de Mayer et al. (1995), "entre la confianza personal y la confianza en las instituciones se debe hablar... sobre todo de la confianza que es imprescindible para el funcionamiento exitoso de una empresa. Se trata aquí de una confianza personal que se distingue de la confianza entre amigos o cónyuges, porque los participantes no exponen toda su existencia a la vulnerabilidad, sino sólo una parte de sí mismos. Cada uno actúa por así decir como sociedad personal de responsabilidad limitada. Y esto modifica también la naturaleza de la confianza. Aquí la confianza no es una convicción elemental inmediata, sino un instrumento conscientemente producido para el éxito empresarial. Se podría decir que se instrumentaliza de modo poco correcto la disposición humanitaria, y eso es a menudo el caso." (Spaemann, 2005:142). 
El riesgo de la instrumentalización vendría dado por una utilización de la confianza en beneficio exclusivamente empresarial. No obstante, aunque reconoce el riesgo que supone la instrumentalización de la confianza, Spaemann subraya la necesidad de ésta más allá de los intereses empresariales. "La confianza de la que vive una empresa, puesto que es una confianza personal, tiene que tener siempre una cierta sobreabundancia sobre el interés empresarial, justo si quiere ser comercialmente ventajosa. Sólo puede cumplir con su función instrumental si hasta cierto punto va más allá de esta función,... Queda fuera de cualquier duda el hecho de que la confianza, si no imprescindible, es de la máxima importancia para la dirección de una empresa (Spaemann, 2005:143).

¿Y cómo evitar que se instrumentalice la confianza? Spaemann dice "No hablaré de medidas para la creación de confianza; para la obtención o, lo que es mucho más difícil, la reobtención de la confianza. Sólo quisiera decir una cosa: todas estas medidas son vanas, se consideran como manipulación y no dan resultado si no contienen el núcleo de aquello que constituye la confianza, que consiste en hacerse realmente vulnerable. Ello no significa vulnerabilidad total y, además, se desarrolla en forma gradual y abierta a la experiencia. Pero el límite de vulnerabilidad que cada uno pone es ciertamente el límite de la confianza. El punto hasta el cual uno está dispuesto a ir no se puede determinar de una vez para siempre" (Spaemann, 2005:146).

Cuando hablamos de confianza resulta imposible elaborar un modelo capaz de predecir este fenómeno humano como si de un fenómeno de la termodinámica se tratara. Al igual que no es posible predecir con total certeza si puedo confiar en alguien, tampoco es exacto reducir la explicación de la generación de confianza exclusivamente a un proceso secuencial previsible. Confiar supone siempre una decisión libre de cada persona y es precisamente esa libertad la que impide encerrar la generación de confianza en un excesivo esquematismo. Si todo fuera previsible y capaz de ser sometido a un proceso lógico, no hablaríamos ya de confianza. Sin embargo, sí es posible establecer modelos que permitan entender mejor el fenómeno, siempre y cuando se reconozcan de modo explícito sus limitaciones, y esto es precisamente lo que pretendemos hacer aquí.

Al subrayar el olvido de las virtudes morales en el modelo propuesto por Mayer et al., queremos incidir precisamente en que uno de los aspectos que permitirá aumentar nuestra confianza hacia alguien y que permitirá reducir el riesgo de equivocarse al confiar es la presencia de virtudes morales en su carácter. Más allá de una visión de la integridad como la que describen Mayer et al, (1995) reducida a la posesión de unos principios, Spaemann hablará del concepto aristotélico de virtud. La persona virtuosa es más digna de confianza. "la virtud...es la capacidad, consolidada por el hábito, de hacer realmente lo que uno ha reconocido como bueno y deseable. Se podría afirmar que la virtud es aquello que capacita a alguien para fiarse de sí mismo. Y sólo aquel que puede fiarse de sí mismo, que puede confiar en sí mismo, justifica también la confianza que otros puedan tener en él." (Spaemann, 2005:139).

La integridad como antecedente de la confianza quedaría incompleta si no recogiera el concepto de virtud. Los principios son necesarios pero "no basta saber qué es lo bueno, y cómo lograrlo, hay que obrar en la práctica y ahí es donde aparece el concepto de virtud" (Guillen, 2006:6).

Para terminar con las aportaciones que, a nuestro entender ofrece el pensamiento de Robert Spaemann al trabajo seminal de Mayer et al. (1995), queremos insistir en la con- 
veniencia de considerar explícitamente la virtud de la prudencia o sabiduría práctica entre los elementos constitutivos de la integridad moral. Se trata de la virtud propia de quienes aciertan en el juicio moral acerca del bien y del mal. Una virtud que sólo puede ser encontrada en personas concretas y que no consiste tanto en tener criterios (principios) como en "tener criterio", esto es, en tener capacidad de juzgar bien en sentido ético.

\section{HACIA UN CONCEPTO MATIZADO DE CONFIANZA EN LAS ORGANIZACIONES}

Tras exponer algunas de las limitaciones que encontramos en el modelo de generación de confianza de Mayer et al. (1995), y habiendo sugerido elementos del pensamiento de Robert Spaemann que mejoran y matizan el modelo, nos proponemos ahora formular un concepto holístico de confianza capaz de recoger estas mejoras. Nuestra propuesta pasa por definir la confianza como:

"una relación entre dos partes de carácter interpersonal y bidireccional, en donde la parte que confía (depositante de confianza) decide libremente hacerse vulnerable ante una acción de la parte en la que se va a confiar (depositaria de confianza). Esta decisión se basa en la expectativa de que ésta última hará bien lo que tenga que hacer sin necesidad de ser controlada".

Como se puede apreciar, proponemos matizar la definición de Mayer et al. (1995), mencionando de forma explicita tres características de la confianza en las organizaciones que no son mencionadas por estos autores: su carácter interpersonal, bidireccional y libre.

Por lo que se refiere a la confiabilidad del depositario de la confianza, ésta se evalúa a partir de tres elementos: la habilidad, la benevolencia y la integridad. Lo que proponemos aquí es una concepción de la integridad ampliada a partir del trabajo de Spaemann y más acorde al pensamiento originario de Aristóteles. Habilidad y benevolencia serían descritas de modo idéntico a como lo hacen Mayer et al. (1995), mientras que la integridad no se reduciría exclusivamente a "los principios que rigen el comportamiento del depositario" y que son compartidos por el depositante de la confianza. Proponemos aquí entender la integridad de un modo más amplio como: "los principios que rigen el comportamiento del depositario, sus virtudes morales y su capacidad para discernir acerca del bien y del mal".

Con estas matizaciones al concepto de confianza nos enfrentamos a las tres limitaciones que han sido descritas más arriba. La primera limitación de corte más teórica, y que se refiere al riesgo de caer en un modelo excesivamente mecanicista, podría ser superada si se reconoce en todo momento el papel de la libertad tanto en el depositante como en el depositario, ya que ambos pueden actuar siempre de modo imprevisible, por razones que ningún modelo podrá recoger. Las otras dos limitaciones (olvido de las virtudes y el juicio moral sobre el bien como partes de la integridad) serían limitaciones del modelo. En este sentido, sería posible revisar la escala de medición de la integridad añadiendo ítems que contemplen los hábitos morales y la habilidad de discernimiento del depositario ${ }^{4}$.

\footnotetext{
${ }^{4}$ El objetivo sería el de revisar e incorporar los ítems que componen la escala para medir la variable integridad, con tal de incorporar las aportaciones de este trabajo validando la fiabilidad del nuevo instrumento de medida.
} 


\section{CONCLUSIONES}

Este trabajo se proponía como objetivo una revisión del modelo de generación de confianza organizativa de Mayer et al. (1995), bajo la perspectiva del humanismo ético de Robert Spaemann. Tras el estudio de la obra de Mayer et al., se detectaron algunas limitaciones en su comprensión del pensamiento aristotélico, citado por estos autores como marco teórico análogo al descrito en su modelo.

Las aportaciones de la obra de Spaemann (2005), y su comprensión más matizada del pensamiento aristotélico, hacen posible superar las limitaciones encontradas y, proponer algunas mejoras al modelo que consistirían en reconocer de modo explícito, en la definición de la confianza, que ésta es una relación interpersonal bidireccional y que es sobre todo libre. Y en considerar, también de modo explicito, el papel de las virtudes morales y de la capacidad de juzgar acerca del bien y el mal entre los elementos que definen la integridad del depositario de la confianza.

Como todo trabajo de investigación, este estudio cuenta con evidentes limitaciones, y más en un campo tan complejo como es la generación de confianza. La principal limitación es sin duda su carácter exclusivamente teórico, si bien es cierto que reflexiones como las realizadas aquí deben ser previas a cualquier intento de contrastación empírica.

Complementariamente, consideramos que este trabajo puede abrir una nueva línea de investigación para el diálogo entre la ética y las ciencias sociales de modo que ambas puedan colaborar en una explicación más completa del fenómeno de la confianza y su generación. Por su parte, la ética y la antropología filosófica permitirán establecer los límites a los que las ciencias sociales no son capaces de llegar en la explicación de los fenómenos humanos, a la par que podrán abrir nuevos horizontes acerca de dimensiones del comportamiento que habían sido olvidadas. A su vez, las ciencias sociales, podrán confirmar y verificar empíricamente aquellas intuiciones de la filosofía que puedan ser verificables.

Tan sólo si se consideran de valor las aportaciones de una y otra disciplina valdrá la pena proponer futuros trabajos empíricos que complementen lo expuesto en esta investigación. Si éste fuera el caso, una clara línea de investigación que se desprende de este estudio sería la de ampliar y validar la escala de medición de la integridad incorporando en ella las virtudes y la capacidad del juicio moral acerca del bien.

Todo este trabajo permitiría seguir adelante con la búsqueda de un concepto de confianza organizativa de carácter humanista y por tanto universal, capaz de ser aplicado en el ámbito de cualquier organización humana. Pensamos que este avance sólo será posible en el marco de un diálogo constructivo, como el que hemos intentado mantener en este trabajo, entre las ciencias humanas y las ciencias sociales.

\section{BIBLIOGRAFÍA}

ARISTOTLE (1992): The Art of Rhetoric Penguin Classic. disponible en www.classics.mit.edu/ aristotle/rhetoric.html.

BUTLER, J. K., JR. (1991): “Toward Understanding and Measuring Conditions of Trust: Evolution of a Conditions of Trust Inventory", Journal of Management, Vol. 17, №. 3, p. 643. 
BUTLER, J. K., JR. y CANTRELL, R. S. (1984): “A behavioral decision theory approach to modeling dyadic trust in superiors and subordinates", Psychological Reports, Vol. 55, No. 1, pp. 19-28.

DEUTSCH, M. (1958): “Trust and suspicion”, Journal of Conflict Resolution, Vol. 2, no. 4, pp. 265279.

GUILLEN, M. (2006): Ética en las organizaciones. Construyendo confianza, Pearson Educación.

JENSEN, M. C. y MECKING, W. H. (1976): "Theory of the firm: Managerial behavior. agency costs and ownership structure", Journal of Financial Economics, Vol. 3, pp. 305-360.

KNOX, R. E. y KEE, H. W. (1970): "Conceptual and methodological considerations in the study of trust and suspicion", The Journal of Conflict Resolution (pre-1986), Vol. 14, no. 3, pp. 357-366.

MARIN-GARCIA, J. A. (2002): La gestión participativa en las grandes empresas industriales españolas: grado de uso, resultados obtenidos y comparación internacional. http://hdl.handle. net/10251/5845

MAYER, R. C. y DAVIS, J. H. (1999): "The effect of the performance appraisal system on trust for management: A field quasi-experiment”, Journal of Applied Psychology, Vol. 84, n 1, pp. 123-136.

MAYER, R. C. y GAVIN, M. B. (2005): "Trust in management and performance: Who minds the shop while the employees watch the boss?", Academy of Management Journal, Vol 48, n5, pp. 874-888.

MAYER, R. C.; DAVIS, J. H. y SCHOORMAN, F. D. (1995): “An integrative model of organizational trust", Academy of Management.The Academy of Management Review, Vol. 20, no. 3, pp. 709-734.

MELE, D. (2000): "Racionalidad ética en las decisiones empresariales", Revista Empresa y Humanismo, Vol. II, pp. 411-437.

MEYER, J. W. (1983): Organizational factors affecting legalization in education, en J. W. Meyer y R. Scott (dir): Organizational environments: Ritual and rationality, pp. 217-232, Jossey-Bass.

SCHOORMAN, F. D. y BALLINGER, G. (2006): Leadership, trust and client service in veterinary hospitals, Working paper, Purdue University, West Lafayette, IN.

SCHOORMAN, F. D.; MAYER, R. C. y DAVIS, J. H. (1996): "Empowerment in veterinary clinics: the role of trust in delegation", in 11th Annual Meeting of the Society for Industrial and Organizational Psychology.

SCHOORMAN, F. D.; MAYER, R. C.; y DAVIS, J. H. (2007). "An integrative model of organizational trust: past, presente, and future". Academy of Management. The Academy of Management Review, Vol. 32, no. 2, pp. 344-354.

SERVA, M. A.; FULLER, M. A. y MAYER, R. C. (2005): "The reciprocal nature of trust: a longitudinal study of interacting teams", Journal of Organizational Behavior, Vol. 26, nº 6, pp. 625-648.

SITKIN, S. B. y BIES, R. J. (1994): “The legalization of organizations: A multitheoretical perspective”, en S. B. Sitkin y R. J. Bies (dir): The legalistic organizations, pp. 19-49, Sage.

SPAEMANN, R. (2005): “Confianza”, Revista Empresa y Humanismo, Vol. IX, pp. 131-148.

WILLIAMSON, O. E. (1975): Markets and hierarchies: Analysis and antitrust implications. New York: Free Press.

ZAND, D. E. (1972): "Trust and Managerial Problem Solving", Administrative Science Quarterly, Vol. 17, n'. 2, pp. 229-239. 
